\title{
Faut-il en finir avec les stéréotypes de sexe ? Revue de questions critique sur les études psychosociales des relations entre sexes
}

\author{
TostaIN Manuel ${ }^{\mathrm{a}}$
}

\footnotetext{
a Université de Caen, Centre d'étude et de recherche sur les risques et les vulnérabilités (EA 3918), France.

Correspondance : Manuel Tostain, Université de Caen, campus 1, UFR de psychologie, bureau SE 611, Esplanade de la paix, 14032 Caen cedex, France.

Courriel : manuel.tostain@unicaen.fr
}

Texte reçu le 19 décembre 2014 et accepté le 5 novembre 2015 .
Résumé : L'auteur examine une tendance, dominante en psychologie sociale des relations entre sexes, à se focaliser sur les stéréotypes de sexe dont seraient victimes les individus, et à privilégier une lecture en termes de domination masculine pour en rendre compte. À travers une analyse critique de travaux très connus dans ce champ d'étude, nous nous attachons à montrer que cette orientation s'associe le plus souvent à une surestimation de l'impact des stéréotypes de sexe et conduit à une vision partielle, voire partiale, des dynamiques entre sexes. Nous souhaitons, par cette revue de question, contribuer à un débat sur la manière problématique dont souvent, selon nous, la psychologie sociale, envisage les relations entre sexes.

Should We Have Done with Sexual Stereotypes? A Review of Critical Questions in Psychosocial Studies on the Relations Between the Sexes

Abstract: We examine a tendency, dominant in the social psychology of relations between the sexes, to focus on sexual stereotypes that affect individuals, and to privilege a reading in terms of masculine domination in order to account for them. Through a critical analysis of well-known works in this field, we seek to show that this orientation is usually associated with an overestimation of the impact of sexual stereotypes and leads to a partial, and even partisan, vision of the dynamics between the sexes. In reviewing this question, we hope to contribute to a debate on the way in which social psychology often envisages relations between the sexes, and which we believe to be problematic. 


\section{INTRODUCTION}

Cet article a pour objectif d'interroger la pertinence d'une tendance dominante en psychologie sociale, dans le cadre des stratégies de réduction des inégalités entre femmes et hommes, à se focaliser sur l'impact des stéréotypes de sexe ${ }^{1}$ dont seraient victimes les individus et à privilégier une interprétation en termes de domination masculine pour en rendre compte ${ }^{2}$ (par exemple Glick, Fiske, 1996 ; Jost, Banaji, Nosek, 2004 ; Lorenzi-Cioldi, 2009 ; Sarlet, Dardenne, 2012 ; Sidanius, Pratto, Van Laar, Levin, 2004).

Le point de vue développé ici est que l'analyse des stéréotypes de sexe, associée à une lecture en termes de domination masculine, pose selon nous quatre types de problèmes. D'abord, cette analyse s'accompagne de biais qui tendent à surévaluer le poids et l'effet des stéréotypes de sexe. Ensuite, cette analyse s'inscrit dans une vision qui nous semble par trop déterministe et mécaniste des rapports entre individus et société, où les dynamiques des individus, en tant qu'acteurs autonomes et décidant de leur propre vie, sont singulièrement non reconnues. Troisièmement, cette analyse alimente

1. Par stéréotype de sexe, on entend les traits et comportements attendus et valorisés pour les membres d'un groupe de sexe donné. Nous parlerons de stéréotypes de sexe et non pas de stéréotypes de genre pour nous situer du côté des acteurs sociaux. Si les stéréotypes résultent d'une construction sociale (et en ce sens renvoient, si on se réfère aux conventions terminologiques de la discipline, au genre, le sexe étant plutôt associé à la dimension biologique), ces stéréotypes, si on se place du point de vue des individus, les visent et les affectent avant tout parce qu'ils sont perçus comme appartenant au sexe biologique masculin ou féminin (pour une discussion des problèmes posés par cette distinction entre sexe et genre, voir Hirata, Laborie, Le Doaré, Senotier, 2000 ; Théry, 2007). Par convention, nous parlerons de groupe féminin pour désigner les filles et les femmes, de groupe masculin pour désigner les garçons et les hommes. Nous mettrons entre guillemets féminin et masculin quand notre propos ne concernera pas directement le sexe des individus mais des dimensions socialement construites et associées, de façon stéréotypée, à tel ou tel groupe de sexe : traits dits « féminins » ou dits « masculins » par exemple.

2. Par domination masculine on entendra le principe selon lequel les hommes tendent à se considérer supérieurs aux femmes et, dans le cadre de leur volonté de domination sur les femmes, disposent d'un pouvoir matériel et symbolique qui limite les comportements et pensées des femmes, celles-ci, pour une large part, par aliénation au point de vue des hommes, ne contestant pas ce pouvoir. Les théories de la domination masculine considèrent que ce principe est présent dans toutes les sociétés, qu'elles soient traditionnelles ou modernes (Bourdieu, 1998). une vision partielle, voire partiale, de la réalité des relations entre femmes et hommes peu à même de rendre compte des évolutions sociétales contemporaines et, notamment, de l'hétérogénéité sociale et psychologique des groupes féminin et masculin. Enfin, cette analyse peut se révéler paradoxalement contre-productive dans la lutte contre les inégalités entre femmes et hommes. Les discours en termes de domination masculine, qui affleurent dans certaines politiques en faveur de l'égalité, peuvent susciter des sentiments d'incompréhension chez les individus, dans la mesure où ils peuvent $\mathrm{y}$ voir la non-reconnaissance de la manière dont ils promeuvent, au quotidien et à leur niveau, l'égalité entre les femmes et les hommes. Dès lors, parce qu'ils ne se reconnaissent pas dans ces discours et images négatives qu'on leur renvoie d'eux-mêmes, le risque est qu'ils prennent de la distance avec ces politiques d'égalité ou qu'ils ne se sentent pas concernés et soient, de ce fait, moins mobilisés sur ces questions. $\mathrm{Au}$ cours de cet article nous nous interrogerons successivement sur la présence de biais évaluatifs liés au sexe, sur la menace du stéréotype de sexe, sur la validité des procédures mesurant l'adhésion aux stéréotypes de sexe et au sexisme et sur les liens supposés entre stéréotypes de sexe et comportements discriminatoires. Enfin, nous terminerons par une discussion critique à propos des postulats qui accompagnent, le plus souvent, les études sur les stéréotypes de sexe. Mais avant de commencer, précisons qu'il ne s'agit pas pour nous de contester que les stéréotypes de sexe, de façon explicite ou par des mécanismes inconscients, puissent avoir des effets négatifs et qu'il convient dans ce sens de les étudier (McConnell, Leibold, 2001). Comme nous tâcherons de le démontrer, nous contesterons néanmoins la généralité de la présence de ces stéréotypes de sexe et l'importance de leurs effets négatifs.

\section{Des apports de la psychologie sociale dans la lutte contre les stéréotypes de sexe et le sexisme ordinaire}

La psychologie sociale, forte d'une longue tradition de recherche sur les stéréotypes (les théories implicites de personnalité, les croyances à l'égard de certaines catégories de personnes ou de groupes), les préjugés (les attitudes, positives ou négatives, à l'égard de ces catégories) et les discriminations (les comportements négatifs à l'encontre de ces catégories), s'est résolument emparée de la question des stéréotypes notamment de sexe et de ses effets (Allport, 1954 ; Bourhis, Leyens, 1998 ; Fiske, 1998 ; Lippmann, 1922). L'objectif est légitime, l'agenda devant à la fois autoriser des avancées sur 
les plans théorique, méthodologique et pratique. Au niveau de la lutte contre les discriminations liées au sexe, la psychologie sociale et ses travaux, sont ainsi au premier plan ${ }^{3}$. Mais que présente-t-on au juste ? Quels sont les postulats sous-jacents ? Y a-t-il une correspondance entre les résultats issus des recherches expérimentales s'inscrivant dans le cadre des théories de la domination et les phénomènes constatés en milieu social «naturel »?

\section{TROIS EXEMPLES DE RECHERCHES CARACTÉRISTIQUES SUR LES EFFETS DES STÉRÉOTYPES DE SEXE}

Nous allons commencer par la présentation de trois recherches de psychologie sociale qui sont révélatrices d'une certaine manière de penser les différences entre sexe. Bien entendu, ce choix est arbitraire si on se réfère aux dizaines de recherches qui ont été publiées sur la question. Si nous les avons choisies, c'est qu'elles ont pour caractéristique d'être amplement rappelées dans les manuels de psychologie sociale, dans les recherches, et dans les campagnes de sensibilisation aux discriminations liées aux stéréotypes de sexe. En ce sens, elles peuvent prétendre faire partie d'une certaine culture commune, à la fois, dans et au-delà de la psychologie sociale (Gergen, 1973). Le message de ces recherches est de nous alerter sur les altérations et déformations de la perception de la réalité que font subir nos préconceptions et stéréotypes de sexe et, en particulier, de souligner les effets insidieux d'une certaine domination masculine. Ces recherches, du fait de leur popularité, présentent l'intérêt d'avoir fait l'objet de réplications. Or, ces réplications sont moins souvent évoquées et leurs résultats donnent à voir une autre image des relations intersexes que le message initial qui émane des recherches princeps.

\section{La recherche de John et Sandra Condry (1976)}

Dans cette recherche, on faisait visionner à des étudiants, hommes et femmes, une vidéo d'un jeune enfant de neuf mois manifestant des émotions face à différents jouets qui lui étaient présentés. Selon les conditions expérimentales, cet enfant était désigné comme une fille (Dana) ou un garçon (David) bien qu'il s'agissait du même enfant. Ensuite, les sujets devaient décrire cet enfant à l'aide d'échelles d'at-

3. On pourra, par exemple, consulter « Discriminations et gestion de l'égalité et de la diversité » de Pascal Tisserant et Richard Bourhis sur le site web Canal U, ou le site <web femmes.gouv.fr $>$ du ministère des Affaires sociales, de la Santé et des Droits des femmes. tribution d'émotions et du différenciateur sémantique d'Osgood (Osgood, Suci, Tannenhaum, 1957). Les résultats montrèrent que lorsque l'enfant était supposé être un garçon, il était perçu comme plus actif, plus «puissant » (potency), qu'il exprimait davantage de colère et moins de peur que lorsqu'il était supposé être une fille. Le message que l'on retient de cette recherche est que les individus sont influencés par les stéréotypes de sexe les plus traditionnels (la force, l'activité pour le garçon versus une relative passivité de la fille, son émotivité craintive), un simple étiquetage arbitraire du sexe de l'enfant suffisant à les mobiliser et à modifier leur perception de la réalité. Signalons d'abord que ces effets d'étiquetage liés au sexe sont loin d'être systématiques. Dès 1989, Stern et Karraker, après avoir analysé 23 recherches sur ce sujet, notent que seuls $18 \%$ des effets d'étiquetage, mesurés dans ces études, étaient significatifs. Ensuite, l'étude de Condry et Condry datant de 1976, on peut se demander si ce type de phénomène reste d'actualité, compte tenu des évolutions sociétales et de l'orientation contemporaine vers davantage d'égalité entre les sexes. Une recherche récente menée par Steuer, Bode, Rada et Hittner (2010) suggère d'y répondre par la négative. Ils ont refait la recherche des Condry en respectant au plus près la procédure initiale. Or, ils ne constatent aucun effet de cet étiquetage de sexe sur les perceptions des sujets : l'enfant, qu'il soit étiqueté garçon ou fille, est perçu de façon similaire aussi bien par les femmes que par les hommes. Il y a, néanmoins, un effet intéressant dans la recherche de Steuer et coll. (2010), les femmes insistant davantage sur la peur de l'enfant, les hommes sur sa colère, ce qui dénote une sensibilité pour partie différente des deux sexes.

\section{La recherche de Kay Deaux et Tim Emswiller (1974)}

Dans cette étude, on présentait à des étudiants-es des enregistrements d'hommes et de femmes en train de réaliser des discriminations perceptives à propos d'objets à connotation «masculine » (par exemple, un cric de voiture) ou "féminine » (par exemple une serpillière (sic)). Les étudiants-es, mis en position d'évaluateurs, devaient indiquer dans quelle mesure la performance des hommes et des femmes relevait de la chance ou de leur compétence. Les résultats montrèrent, aussi bien chez les étudiants que chez les étudiantes, que la réussite à la tâche à connotation « masculine » était davantage attribuée à la compétence quand elle était réalisée par un homme que par une femme, tandis que la réussite à la tâche à connotation «féminine » était autant attribuée à la compétence, que cette tâche fût 
réalisée par un homme ou par une femme. Autrement dit, il n'y a pas de symétrie évaluative, l'homme faisant l'objet d'une valorisation plus grande que la femme dans le domaine "masculin », équivalente à celle de la femme dans le domaine " féminin ». Et, fait important, dans la mesure où il n'y a pas d'effet du sexe des évaluateurs (les étudiants et les étudiants répondent de la même façon), cette asymétrie en faveur des hommes est intégrée par les femmes elles-mêmes, qui, ainsi, se portent préjudice. On interprète ce résultat en considérant qu'il s'explique par le statut plus élevé et la domination des hommes dans notre société. Mais, comme si cela ne suffisait pas, quand on rapporte cette expérience, on accentue encore ce phénomène : concrètement, on déclare que cette recherche montre que le succès d'une performance est expliqué, pour un homme, par la compétence, pour une femme, par la chance (par exemple Légal, Delouvée, 2008), ce qui revient à surinterpréter ce qui est constaté dans l'expérience. Ainsi, les préconceptions influencent aussi parfois les chercheurs ${ }^{4}$. Cela dit, ce qu'il faut mentionner, c'est que dès le début des années 1980, diverses recherches sur cette question de l'attribution de compétences n'ont pas retrouvé ces résultats, voire ont parfois obtenu des résultats inverses : davantage de compétence attribuée à la femme qu'à l'homme (Haccoun, Stacy, 1980 ; Lips, Myers, 1980 : Smith, Whitehead, Sussman, 1984). Post montrera, en 1981, que cet effet asymétrique négatif à l'encontre des femmes dépend notamment des orientations politiques : par exemple les femmes « libérales » (au sens américain « d'opinions ouvertes sur le plan des mœurs »), contrairement aux femmes conservatrices, ne manifestent pas ce biais. Enfin, une méta-analyse de Swim et Sanna (1996) conclura que ces effets d'attribution, liés au sexe des sujets, sont généralement faibles.

Mentionnons, dans le même ordre d'idée, que les résultats de la recherche souvent citée de Goldberg (1968), mettant en évidence, chez des femmes, qu'un article supposé écrit par une femme est moins bien évalué que ce même article supposé avoir été

4. Il faut dire, à la décharge des commentateurs de cette expérience, que cette exagération se trouve déjà dans le titre de l'article de Deaux et Emswiller (1974). Plus généralement, à propos des biais liés aux préconceptions des chercheurs, on sait que, même dans un cadre expérimental rigoureux, ces préconceptions peuvent affecter les résultats (Orne, 1962 ; Riecken, 1962 ; Rosenthal, 1964), certaines recherches montrant même que les compères des expérimentateurs, pourtant non dupes par définition, sont eux aussi influencés par ce qu'ils sont amenés à faire croire aux sujets naïfs (Alaphilippe, 1986 ; Laurens, Moscovici, 2005). écrit par un homme, seront également rapidement mis en cause. Ainsi, dès 1975, Levenson, Burford, Bonno et Davis qui répliqueront cette recherche sur une population féminine mais aussi masculine, ne constateront pas ce biais, le sexe de l'auteur de l'article n'affectant pas son évaluation ou, s'il l'affecte, c'est alors dans le sens d'une meilleure évaluation des articles supposés écrits par des femmes. Une méta-analyse de Swim, Borgida, Maruyama et Myers, (1989) confirmera cette absence de biais. Une synthèse réalisée par Eagly, Mladinic et Otto (1991) mettra, en outre, en évidence que les femmes, en termes de traits de personnalité, de désirabilité sociale de ces traits, sont généralement mieux évaluées que les hommes, aussi bien par les femmes que par les hommes. C'est ce que ces auteurs appelleront le «Wonderful women effect» (l'effet « Les femmes sont merveilleuses ») (Eagly, Mladinic, 1994). Cet effet « Les femmes sont merveilleuses » peut s'expliquer par le fait que des dimensions positives (telles que l'attention aux autres, la sensibilité) sont vues comme plus fréquentes chez les femmes que chez les hommes et, qu'à l'inverse, des dimensions négatives (telles que l'agressivité ou l'égoïsme) sont perçues comme plus fréquentes chez les seconds que chez les premières (Gilligan, 1986).

Pour terminer sur ces études sur les stéréotypes, nous présenterons une recherche française, très utilisée dans la sensibilisation aux stéréotypes de sexe, qui pose la question de l'actualité du phénomène observé en laboratoire au regard de la réalité sociale.

\section{La menace du stéréotype (de sexe)}

On sait depuis longtemps que faire partie d'un groupe qui est la cible de stéréotypes négatifs peut avoir des effets délétères sur nos performances (Katz, Epps, Axelson, 1964). C'est ce que préciseront Steele et Aronson (1995) avec la notion de menace du stéréotype, où la peur de confirmer un stéréotype, même si on le sait infondé, en créant une tension et une charge mentale supplémentaire, amène finalement le sujet qui en est la cible à le

5. Précisons que ce phénomène favorable aux femmes sera vérifié en utilisant des procédures dans lesquelles les évaluations des cibles féminines et masculines sont réalisées séparément et par des sujets différents (procédure inter-sujets), ceci pour éviter des biais de désirabilité sociale. On sait, en effet, que dans le cadre d'évaluations de cibles des deux sexes par les mêmes sujets (procédure intra-sujet), les sujets peuvent être amenés, pour ne pas donner l'impression de stigmatiser la cible féminine dont le groupe peut subir des inégalités, à mieux l'évaluer que la cible masculine. 
valider ${ }^{6}$. Précisons que c'est dans les situations de confrontation entre groupes, où les stéréotypes sont rendus plus saillants, que cette menace est tout particulièrement mise en évidence. Signalons également un contresens à ne pas faire. Les recherches sur cette menace du stéréotype ne démontrent pas que ce sont les stéréotypes négatifs qui expliquent à eux seuls les moindres résultats des individus qui appartiennent à des groupes qui en sont la cible (Sackett, Hardison, Cullen, 2004). Ce que ces recherches mettent en évidence, c'est que les stéréotypes négatifs ajoutent des difficultés supplémentaires pour les groupes défavorisés, mais les causes centrales relèvent principalement des conditions sociales des individus appartenant à ces groupes. Quand ces conditions sociales s'égalisent entre groupes sociaux initialement de statuts différents, quand l'environnement ou le contexte sont plus égalitaires, les différences de performance entre ces groupes tendent à disparaître, cette menace du stéréotype devenant aussi moins agissante (Bonnot, Croizet, 2011 ; Croizet, Claire, 1998 ; Croizet, Dutrévis, 2004).

Au niveau des stéréotypes de sexe, Pascal Huguet et Isabelle Régner $(2007,2009)$, vont mettre en évidence un phénomène similaire à celui étudié par Steele et Aronson (1995). Dans leurs recherches, ils visaient à étudier l'effet du stéréotype négatif selon lequel les filles sont moins bonnes que les garçons en mathématiques, la menace de ce stéréotype pouvant pour partie expliquer pourquoi les jeunes filles auraient de moindres résultats dans cette matière que les garçons, et pourquoi elles se dirigent moins dans des carrières scientifiques ${ }^{7}$. Dans ce cadre, ils font passer à des collégiens et à des collégiennes français, placés en groupes mixtes, un test, soit censé mesurer leur aptitude en géométrie (mobilisant donc implicitement ce stéréotype négatif pour les filles) soit censé mesurer leur mémoire ou leur capacité en dessin (dimensions jugées non attachées à un stéréotype de sexe). Conformément aux hypothèses, les filles réussissent moins bien que les garçons quand l'épreuve est associée au stéréotype négatif (géométrie). Par contre, elles réussissent

6. Précisons que l'on peut provoquer aussi l'effet contraire (meilleures performances) quand on active un stéréotype positif attaché au groupe auquel appartient l'individu qui doit réaliser la tâche (phénomène de « stereotype boost » ou « coup de pouce du stéréotype » (Shih, Pittinsky, Ho, 2012).

7. Pour une synthèse des travaux mettant en avant l'effet de la menace de ce stéréotype de sexe, voir Régner, Steele, Ambady, Thinus-Blanc, Huguet (2014). mieux que les garçons quand cette épreuve n'est pas associée à ce même stéréotype (épreuve de mémoire et de dessin), ce dernier phénomène restant pour partie à expliquer. Ainsi, pour ne retenir que le résultat à l'épreuve de géométrie, cette étude met en évidence, en situation de mixité, les effets négatifs d'un stéréotype de sexe sur les performances des filles. Concernant cette menace du stéréotype liée aux mathématiques, il convient cependant de noter qu'une méta-analyse récente portant sur 31 recherches publiées et complétées par trois expériences sur 931 élèves américains âgés de 9 à 17 ans, conclut à l'absence d'effet significatif de la menace de ce stéréotype (Ganley, Mingle, Ryan, Ryan, Vasilyeva, Perry, 2013). D’un autre côté, ces effets négatifs sont-ils nécessairement présents dans la vie réelle au niveau des résultats scolaires ? Si cette menace de ce stéréotype joue un rôle, on devrait a minima constater que dans le contexte des écoles mixtes filles/garçons, les filles sont effectivement moins bonnes que les garçons en mathématiques. Or, ce n'est pas le cas. Aux États-Unis, une méta-analyse de Lindberg, Hyde, Petersen et Linn (2010) montre que les filles et les garçons ont des performances similaires dans cette matière. C'est également vrai en France où les statistiques du ministère de l'Éducation nationale (2013) mettent en évidence que les filles ont globalement un niveau équivalent à celui des garçons en mathématiques. Ajoutons que dans le baccalauréat scientifique par excellence, à savoir le bac $\mathrm{S}$, où elles représentent $45 \%$ des effectifs, les filles tendent même à avoir de meilleurs résultats que les garçons. On peut donc s'interroger sur la présence et la signification de cette menace d'un stéréotype dont l'effet ne se manifeste pas nécessairement tant dans les recherches expérimentales que quand on la confronte à l'épreuve des situations réelles de mixité scolaire. Dès lors, plutôt que de s'axer, dans les campagnes d'information en faveur de l'égalité entre sexes, sur la menace de ce stéréotype à la réalité discutée, ne faudrait-il pas mieux contester certaines idées reçues en soulignant ce résultat : les filles sont aussi bonnes en mathématiques que les garçons. Des recherches récentes montrent d'ailleurs les effets paradoxaux liés à l'omniprésence de ce discours nous alertant sur les méfaits de ce type de stéréotypes (Breda, Ly, 2014). Par exemple Terrier (2014), dans sa recherche qui portait sur 3964 collégiens et collégiennes français de $6^{\mathrm{e}}$, constate que les enseignants en mathématiques, inconsciemment, surnotent les filles par rapport aux garçons (alors que les évaluations standardisées et anonymes - et donc sans connaissance du sexe de l'élève - attestent du niveau équivalent des filles et des garçons). Ainsi, ces enseignants 
pratiquent une discrimination en faveur des filles, de peur, peut-être, d'être les acteurs involontaires de l'idée commune qui déclare que les filles sont défavorisées par rapport aux garçons dans les disciplines scientifiques. Autrement dit, le stéréotype « les filles sont moins bonnes en mathématiques » ressemble ici à une prophétie auto-réalisatrice mais dans le sens contraire de ce qui est habituellement entendu et attendu.

Pour aller plus loin, rappelons que les individus, contrairement à ce que tendent à suggérer certains travaux sur la menace du stéréotype, ne sont pas nécessairement les victimes de cette menace. Ainsi, quand on présente explicitement à des femmes (et non pas implicitement, comme c'est le cas dans la plupart des recherches sur la menace du stéréotype) un stéréotype négatif qui caractérise leur groupe (par exemple en leur déclarant qu'elles sont moins bonnes négociatrices que les hommes), on note non pas une diminution mais, au contraire, une augmentation de leurs performances (Kray, Thompson, Galinsky, 2001). On peut expliquer ce résultat par un phénomène de réactance ou comme la réaction à un sentiment d'injustice : les femmes mobilisent leurs ressources cognitives pour récuser un stéréotype qui les figent dans une identité négative. On peut d'ailleurs constater que lorsqu'on met les femmes dans des situations de maîtrise et d'autonomie, ce type de menace du stéréotype s'estompe également (Van Loo, Rydell, 2013).

En définitive, dans ces recherches princeps que l'on trouve dans de nombreux manuels actuels de psychologie sociale, le message est de nous dire que nous sommes victimes des stéréotypes de sexe. Certes, bien entendu, des effets négatifs des stéréotypes de sexe peuvent avoir cours, mais ce message tel qu'il s'énonce est éminemment discutable. Ainsi qu'on a pu le noter, les données issues tant de certaines réplications que des pratiques sociales en milieu « naturel » ne corroborent pas toujours, loin de là, cette assertion. Dans ce sens, l'explication de certaines différences de comportement entre filles et garçons en termes uniquement de stéréotypes de sexe nous paraît réducteur, comme c'est le cas quand on cite les différences de choix de filières entre les unes et les autres. S'il est vrai que les filles se dirigent moins que les garçons dans les filières scientifiques, il faut ajouter que les garçons aussi s'y destinent moins, ces filières connaissant une baisse d'effectifs générale. Dès lors, ce qui s'y joue ce n'est pas uniquement une question de stéréotype de sexe mais aussi une baisse de désirabilité sociale de ces filières qu'il s'agit d'interroger. Il faut ajouter que cette désaffection des jeunes filles ne touche que certaines matières scientifiques (physique, chimie fondamentale) et qu'elles sont plus nombreuses que les garçons dans d'autres matières scientifiques (biologie, médecine), ce qui cadre assez mal avec une lecture en termes de stéréotype de sexe, sauf à considérer que ces matières ne sont pas scientifiques ou qu'il existe maintenant une bi-catégorisation de ces matières, certaines jugées plus féminines, d'autres plus masculines. Néanmoins, même dans cette dernière hypothèse, cela montre que le champ du possible en matière scientifique s'est singulièrement élargi pour les filles.

À ce stade, on peut se poser la question de la validité externe des résultats issus de recherches expérimentales souvent réalisées en laboratoire, de leur généralisation à la vie « réelle ». Ne devrait-on pas s'assurer, parallèlement à la réalisation de telles expériences, que les mécanismes étudiés renvoient effectivement à des phénomènes attestés au niveau des pratiques sociales?

Cela dit, des modèles tels ceux de la jugeabilité sociale et de la déterminabilité sociale peuvent se révéler fructueux pour comprendre les conditions dans lesquelles les stéréotypes peuvent ou non s'appliquer. Ces modèles montrent que les influences sociales relatives à certains stéréotypes ne se réalisent que dans certaines conditions. Ce serait le cas quand la personne en position de juge potentiel dispose non seulement d'une information utilisable pour construire son jugement mais aussi qu'elle estime que les paramètres de la situation lui permettent de faire ce jugement (jugeabilité sociale). Ce serait le cas aussi quand les caractéristiques de la situation sociale induisent une influence sur celui qui est amené à juger (déterminabilité sociale) (Morchain, Schadron, Saint-Bauzel, Béchu, 2013 ; Sabatier, Schadron, Milhabet, Priolo, 2010 ; Schadron, 2005).

\section{DE LA MESURE DES STÉRÉOTYPES DE SEXE ET DU SEXISME ORDINAIRE}

On s'attachera à montrer, dans cette partie, que les modalités de mesure tendent à orienter vers une surestimation du poids des stéréotypes de sexe et du sexisme ordinaire.

\section{Les méthodes de mesure explicite des stéréotypes de sexe et du sexisme ordinaire}

Par mesure explicite, on entend les méthodes dans lesquels il est demandé au sujet d'indiquer son degré d'accord avec des propositions associées à des stéréotypes de sexe. 
La plus connue de ces mesures est le Bem Sex Role Inventory (BSRI) ou Inventaire des rôles de sexe de Sandra Bem (1974). Ce questionnaire est constitué de 60 items renvoyant à des traits de personnalité : 20 items dits « féminins », 20 dits « masculins » et 20 « neutres » (non associés aux rôles de sexe). Il reposait, à l'époque de sa création, sur une vraie innovation théorique, la féminité et la masculinité n'étant, désormais, plus envisagées comme deux dimensions situées sur les bords opposés d'un même continuum (si vous étiez très " féminine " vous étiez de facto peu «masculine »), ce qui était le cas dans les questionnaires plus anciens (par exemple Gough, 1957), mais comme deux dimensions indépendantes. Il autorisait ainsi davantage de liberté dans l'évaluation de la féminité/masculinité des individus. Il faut noter le caractère désormais désuet ou très traditionnel de certains items de ce questionnaire : ainsi, comme items "féminins » on trouve : «crédule », « sensible à la flatterie », «timide » ou comme items « masculins » : « autoritaire », « agit en chef », « dominateur $»^{8}$. Il est un point qui pose question, c'est que les réponses des sujets (tâche d'auto-description) sont souvent interprétées comme la mesure de leur adhésion aux stéréotypes de sexe (Bem, 1981). Est-ce nécessairement le cas ? Imaginons une femme résolument féministe qui marque pleinement son accord avec certains items «féminins » tels « sensible aux besoins des autres » ou « aime les enfants ». $\mathrm{Ne}$ fait-elle qu'adhérer à ces stéréotypes de sexe ? Ne peut-on pas y voir un engagement personnel mettant en avant la priorité qu'elle accorde à la relation à autrui ? Faute, souvent, d'informations complémentaires sur la personne qui a rempli le questionnaire, il est parfois impossible de répondre à la question. Le risque, en raisonnant en termes d'adhésion aux stéréotypes de sexe, est d'appliquer sur le niveau individuel (la tâche d'auto-description), le niveau d'analyse groupal (les stéréotypes de sexe) comme si le second rendait compte mécaniquement du premier (Locksley, Colten, 1979).

Dans ces méthodes de mesure explicite, se sont également développés des questionnaires de sexisme moderne (« néo-sexisme », « sexisme ambivalent») qui mesurent des stéréotypes potentiellement discriminatoires à l'encontre de l'autre groupe de sexe. Ces questionnaires partent de l'idée que le sexisme, en tant qu'expression de la domination masculine,

8. Pour plus de détails sur ce type de questionnaire, voir Gana (1995), K’Delant, Gana (2009), Fontayne, Sarrazin, Famose (2000). Pour une critique du BSRI, voir Choi, Fuqua, Newman (2008). prend des formes plus subtiles car il devient difficile socialement d'attaquer de manière frontale le principe d'égalité entre les sexes. À côté d'un sexisme traditionnel, caractérisé par des attitudes négatives, hostiles à l'encontre des femmes (vues comme agressives, manipulatrices et incompétentes), se développerait un sexisme «bienveillant» qui, sous couvert de mettre en avant des qualités positives propres aux femmes (la pureté, l'attention aux autres) et en avançant des conceptions chevaleresques ou romantiques, les riveraient dans une situation de subordination (en insistant sur leur fragilité, leur besoin d'être protégées par les hommes). Ce second sexisme, étant moins visible, serait plus insidieux et aurait des effets plus négatifs sur les femmes que le sexisme hostile. Les femmes auraient du mal à le contester ou ne seraient pas très conscientes de ses effets pervers. On peut néanmoins s'interroger sur cette idée que le sexisme moderne serait plus redoutable que le sexisme traditionnel car plus insidieux. Rappelons que la situation des femmes dans certaines sociétés traditionnelles, en raison du sexisme traditionnel, est plus inquiétante que la situation des femmes dans nos sociétés occidentales qui seraient caractérisées par un certain sexisme bienveillant. De plus, il nous semble, mais cela n'engage que nous, que traiter les femmes d'incompétentes ou de manipulatrices (sexisme traditionnel) est plus problématique que de les traiter de sensibles mais fragiles (sexisme bienveillant). Cela dit, ces questionnaires reposent sur le postulat que la domination masculine est toujours aussi présente dans nos sociétés modernes, que les changements qualitatifs de cette domination ne modifient pas quantitativement cette domination (Bourdieu, 1998). C'est ce qui fera écrire à des auteurs représentatifs de ce courant, que «plus ça change, plus c'est pareil » (Tougas, Brown, Beaton, Joly, 1995). Au vu des évolutions sociétales de ces trente dernières années vers plus d'égalité, au vu de l'investissement massif des femmes dans le travail, et bien que nombre d'avancées restent fragiles, ce postulat nous paraît très contestable. Mais passons au point qui nous intéresse. On note que le codage des réponses dans ces questionnaires donne singulièrement l'impression de systématiser artificiellement la présence du sexisme et d'en exagérer ainsi la fréquence dans la population masculine. Là encore, comme pour les recherches vues précédemment, nous allons volontairement nous limiter en ne prenant en compte que trois exemples qui, à défaut évident d'exhaustivité, nous semblent révélateurs d'un certain mode de pensée propre à ces questionnaires. Le premier exemple sera tiré du questionnaire de néo-sexisme de Tougas et coll. (1995). On y trouve l'item : «Il est difficile pour une femme de travailler 
en tant que patron ». Une réponse affirmative est notée comme une réponse sexiste. En codant de cette manière cela revient à supposer que la réponse exprime nécessairement l'opinion selon laquelle il n'est pas bien qu'une femme soit patron. Or, il peut s'agir de l'expression d'un point de vue bien différent et qui ne remet pas en cause la possibilité pour une femme d'être patron : à savoir qu'en raison de certaines inégalités qu'il s'agit de combattre, il est plus difficile pour une femme que pour un homme d'être en position de patron, observation que l'on peut étayer par des chiffres faciles à trouver en France : seuls $17 \%$ des dirigeants d'entreprises sont des femmes (Chiffres-clés de l'égalité 2011. Ministère des droits des femmes, de la ville, de la jeunesse et des sports). Les deux exemples suivants seront pris cette fois dans le questionnaire de sexisme ambivalent de Glick et Fiske (1996) qui a été adapté en français par Dardenne, Delacollette, Grégoire et Lecocque (2006) 9. Soit l'item suivant « les hommes sont incomplets sans les femmes ». Une réponse positive est notée comme du sexisme. En effet, pour les auteurs, si les sujets considèrent que les hommes sont incomplets sans les femmes, c'est parce qu'ils pensent que les uns et les autres sont différents et qu'on a besoin de cette différence. Or, toujours pour ces auteurs, affirmer le principe d'une différenciation entre les sexes, notamment dans le cadre de l'hétérosexualité, c'est faire preuve d'une position sexiste de domination masculine. Peut-on dire que c'est le cas ? Le principe d'un questionnaire étant de répondre généralement au regard de soi, imaginons, hypothèse assez fréquente statistiquement, que le sujet qui répond est hétérosexuel : ne peut-il pas considérer, au vu de son orientation sexuée et sexuelle, que le fait de partager sa vie avec une femme soit une condition importante pour son équilibre psychologique ? $\mathrm{Ne}$ peut-on aussi penser qu'une réponse positive à cet item «les hommes sont incomplets sans les femmes » est une manière de rejeter le contraire de l'item ( " les hommes sont complets sans les femmes »), qui sous-tendrait, cette fois-ci une vision sexiste des relations femmes/hommes ? Troisième exemple : soit l'item « les femmes, comparées aux hommes, ont tendance à faire preuve d'un plus grand sens moral ». Une réponse affirmative est encore codée comme du sexisme ( « sexisme bienveillant » en l'occurrence). La justification avancée par les

9. Ce questionnaire connaît une popularité certaine. L'article dans lequel il est présenté est l'un des plus cités d'après le site web de L'Année psychologique, revue où il est paru. Dans un cadre théorique similaire, on pourra consulter l'échelle de justification du système spécifique au genre de Verniers et Martinot (2015). auteurs est que ce type de réponse, en différenciant les femmes des hommes, exprime une tendance à essentialiser les qualités " féminines », à cantonner les femmes dans des traits sexués stéréotypés ${ }^{10}$. Or, on peut aussi avancer que si un sujet répond de cette façon c'est parce qu'il considère qu'en raison de la confrontation à plus d'injustice que les hommes, les femmes peuvent être amenées à développer une plus grande attention morale ${ }^{11}$. Si ce codage est orienté c'est que ce questionnaire repose, à notre avis, sur une double assimilation contestable. Première assimilation : l'hétérosexualité est envisagée implicitement comme un hétérosexisme. Pour défendre ce point de vue, Glick et Fiske (1996) soulignent que, d'un point de vue anthropologique et historique, l'hétérosexualité est systématiquement associée au patriarcat, au paternalisme et, de là, à la domination masculine. On fera remarquer que c'est mettre de côté l'idée que l'hétérosexualité peut se vivre de manière très différente selon les individus : certes, pour certains, cela peut renvoyer à des attitudes dominatrices mais, pour d'autres, cette hétérosexualité peut s'inscrire dans un rapport respectueux voire valorisant à l'égard de la femme (Singly, 2005 ; Kaufman, 2012) ${ }^{12}$. La seconde assimilation consiste à lier différences entre sexes et domination. Pour ces auteurs, la mise en valeur de différences entre les sexes serait à l'origine d'inégalités entre les sexes. En distinguant les deux sexes, en soulignant d'éventuelles qualités distinctives pour les femmes (d'origine innée ou construites socialement, et fussentelles positives comme l'attention aux autres), on assignerait les femmes à des domaines spécifiques (la sphère intime-privée, les professions centrées sur le social, etc.) qui les empêcherait d'accéder à des sphères masculines parfois plus valorisées socialement (les postes à responsabilité, les activités dans le secteur concurrentiel, etc.). Ici, mentionnons trois

10. Rappelons, à titre indicatif, que l'essentialisme n'est pas nécessairement une posture stigmatisante, certains mouvements homosexuels ou transsexuels légitimant leur orientation sexuée en évoquant une disposition d'origine biologique, une essence intime (Castel, 2013).

11. Pour ne pas surcharger le texte, nous n'avons pas multiplié les exemples, mais précisons que près d'un tiers des items de l'échelle peut donner lieu à une analyse critique telle que nous venons de l'esquisser.

12. Mentionnons que ce type de posture théorique, qui tend à assimiler hétérosexualité et hétérosexisme, est politiquement problématique. En effet, dans le débat français actuel sur « La » théorie du genre, elle donne paradoxalement des arguments à tout un courant réactionnaire qui dénonce, dans les mesures sur l'égalité à l'école, une volonté insidieuse de supprimer les différences entre les sexes et de contester l'hétérosexualité (Revue Esprit, 2013). 
points. D'une part, ainsi que l'indique Fraisse (1996), la différence n'est pas le contraire de l'égalité mais de l'identique (ou de l'indifférence). La différence, où la complémentarité entre sexes sur certains aspects, n'implique pas logiquement, nécessairement l'inégalité (Théry, 2007). D'autre part, rappelons que la valorisation des différences entre femmes et hommes ou le combat de ces différences ne constitue pas une ligne de partage claire entre positions antiféministes et positions féministes (Bourcier, Moliner, 2012). Ainsi, certain-es féministes sont, dans une logique universaliste, pour l'indifférenciation des genres (Beauvoir, 1949 ; Badinter, 2003), d'autres, au contraire, revendiquent et valorisent, dans une perspective différentialiste, l'altérité entre les femmes et les hommes (Fouque, 2015 ; Irigaray, 1992). Enfin, ajoutons que les attitudes chevaleresques ou romantiques dénoncées comme du sexisme bienveillant peuvent relever d'une lecture bien différente. Ainsi Claude Habib (2006) montre qu'historiquement, ces attitudes qui se sont développées au travers de cette figure (surannée ?) qu'est la galanterie à la française, ont été, contrairement à certaines idées reçues, un vecteur d'amélioration de la condition féminine ${ }^{13}$.

Si nous contestons la généralité de la présence du sexisme, il ne s'agit pas de nier les effets délétères du sexisme. Des auteurs tels que Dardenne, Dumont et Bollier (2007) ont ainsi mis en évidence des effets négatifs du sexisme bienveillant sur les performances des femmes. Cependant, dans ce type de recherche, il conviendrait, ce qui n'est pas toujours très clairement fait, de distinguer plus systématiquement le sexisme bienveillant proprement dit (quand celui-ci est effectivement une attitude consistant à inférioriser les femmes), de la bienveillance pour les femmes qui n'implique pas, selon nous, de volonté d'infériorisation, et, enfin, les pratiques égalitaristes volontaristes « bienveillantes » telle que la discrimination positive où on privilégie, à compétence égale, une femme par rapport à un homme. Ces trois attitudes, dans la mesure où elles relèvent de dynamiques différentes, peuvent avoir des effets différents. Notons, pour terminer, que certains effets avancés du sexisme font l'objet de discussion. Par exemple, si Brandt (2011) conclut, à partir d'une étude réalisée sur 57 pays, que le niveau de sexisme accrô̂t le niveau

13. Il nous semble, d'ailleurs, que ce n'est pas parce qu'on valorise chez une femme, par exemple, son éventuelle sensibilité (sexisme bienveillant) que cela limite nécessairement ses possibilités d'investir des domaines professionnels variés. d'inégalité entre sexes dans les sociétés, Ullrich et Schlüter (2012) montrent que cet effet devient non significatif si on exclut un pays tel que la Suisse de l'échantillon, ce qui limite singulièrement la portée du résultat de Brandt.

Passons maintenant aux méthodes implicites qui, du fait d'une plus grande technicité de leurs procédures, sont censées permettre de mesurer plus finement les stéréotypes et d'en constater les effets.

\section{Les méthodes implicites de mesures des stéréotypes de sexe : l'exemple de l'IAT de genre (Gender Implicit Association Test)}

Pour éviter certains biais propres aux questionnaires de mesure explicite - les sujets ne souhaitent pas nécessairement révéler ce qu'ils pensent véritablement ou ne sont pas toujours capables de le fairese sont développées des mesures implicites. Ces mesures sont dites implicites dans la mesure où elles sont censées pouvoir révéler les attitudes cachées et véritables des sujets qui peuvent les influencer à leur insu. Par exemple, une méthode très utilisée est basée sur les temps de réaction. Dans cette méthode on présente, généralement sur un écran d'ordinateur, des mots que l'on doit associer à des catégories plus générales. Concrètement un mot apparaît sur l'écran et vous devez appuyer le plus rapidement possible sur une touche, par exemple de la droite du clavier, si ce mot relève par exemple du masculin (le mot « frère »), sur une touche de la gauche du clavier, si ce mot relève par exemple du féminin (le mot « sœur »). Différents mots sont ainsi successivement présentés et vous devez à chaque fois faire la bonne association correspondante. Le postulat de base est de considérer que plus vous faites l'association rapidement et plus cela révèle une tendance personnelle, profonde et vraie. Or, la mise en œuvre de la mesure peut conduire à des résultats discutables ${ }^{14}$. Prenons l'exemple d'un des tests les plus connus, le TAI des stéréotypes de sexe (Test des associations implicites, en anglais IAT, Greenwald, McGhee, Schwartz, 1998). La procédure se déroule en quatre phases. Dans la phase 1, vous devez associer des mots à leur domaine correspondant (par exemple, si le mot «mathématiques » apparaît sur l'écran, l'associer à la catégorie « sciences » en pressant une touche du clavier, ou si c'est le mot 《littérature » l'associer à la catégorie « lettres » en pressant une autre touche du clavier). Dans une phase 2, il s'agit de faire des associations sexuées

14. Pour plus de détails sur ces tests de mesure implicite et les problèmes qu'ils soulèvent, voir Blaison, Chassard, Kop, Gana (2006). 
(par exemple si le mot « père » apparaît, l'associer au domaine «masculin », si c'est le mot « sœur» l'associer au domaine « féminin »). Dans la phase 3 , on vous demande de faire des associations qui relèvent des stéréotypes de sexe (par exemple si les mots « mathématiques » ou « frère » apparaissent les associer à un domaine qui englobe "sciences » et « masculin », si c'est les mots « humanités », ou « tante » les associer à un domaine comprenant « lettres » et « féminin »). Enfin, dans une dernière phase, il est demandé de faire des associations qui vont à l'encontre des stéréotypes de sexe : dans ce cas les mots doivent être associés soit à un domaine regroupant «sciences » et « féminin » (par exemple si les mots « mère » ou « astronomie » apparaissent), soit à un domaine regroupant « lettres » et «masculin» (par exemple si ce sont les mots « humanités » ou « oncle » qui apparaissent). Pour mesurer votre degré d'influence par les stéréotypes de sexe, on calcule la différence de temps de réaction entre la phase 3 (tâche d'association en correspondance avec les stéréotypes de sexe) et la phase 4 (tâche d'association allant à l'encontre des stéréotypes de sexe). Si votre temps de réaction est en moyenne supérieur en phase 4 par rapport à la phase 3 on en conclura que vous êtes influencé par les stéréotypes de sexe et cette influence sera jugée d'autant plus grande que cette différence est importante. Ce type de conclusion paraît néanmoins délicat. En effet, la procédure consistant à mobiliser les stéréotypes de sexe dans la phase 3 , peut déteindre sur la phase 4 et la rendre plus difficile à réaliser (en termes de temps de réaction) dans la mesure où, dans cette nouvelle phase, il faut faire les associations inverses de celles qui étaient demandées dans la phase précédente. Ensuite, les stéréotypes de sexe faisant partie de l'univers social, il paraît assez probable qu'ils soient facilement accessibles, disponibles en mémoire, en tout cas plus accessibles que des exemples contrasexués. Les temps de réaction plus rapides quand l'association va dans le sens des stéréotypes de sexe (et moins rapides quand l'association va à l'encontre des stéréotypes de sexe) peuvent donc renvoyer, non pas nécessairement à l'adhésion ou à l'influence des stéréotypes, mais à leur plus grande fréquence dans la mémoire sociale des individus. Dans ce sens, pour Karpinski et Hilton (2001) l'IAT, contrairement aux mesures explicites des stéréotypes, qui mesureraient vraiment l'adhésion aux stéréotypes, l'IAT, plutôt que de mesurer les attitudes personnelles, révélerait l'environnement culturel des individus. C'est ce qui expliquerait, d'après eux, les liens souvent faibles entre IAT et mesures explicites : pour le genre, si on se réfère à Greenwald, Poehlman, Uhlmann et
Banaji (2009), ce lien entre mesures explicites et implicites est de 0,172. Dans le même sens, Lynott, Connell, O'Brien et Kansal (2012) avancent, suite à leur recherche, que les résultats à l'IAT refléteraient davantage la fréquence linguistique des stéréotypes que l'adhésion des sujets à ces stéréotypes. Il y a donc un débat entre ceux qui pensent que l'IAT révèle l'attitude personnelle des sujets (par exemple, Greenwald et coll., 2009) et ceux qui considèrent, au contraire, que l'IAT reflète les connaissances culturelles des sujets, mais pas nécessairement leurs attitudes personnelles (par exemple Arkes, Tetlock, 2004 ; Tetlock, Mitchell, 2008).

Ce débat se prolonge à propos du caractère prédictif des résultats obtenus avec les tests explicites ou implicites. Le fait d'avoir un score élevé à ces tests est-il l'indice que le sujet présente des comportements discriminatoires effectifs ? D'un côté, Greenwald, Banaji et Nosek (2015), bien que la méta-analyse qu'ils ont effectuée révèle des liens faibles entre les résultats à ces tests et comportements discriminatoires (pour le genre de 0,181 pour les associations explicites et de 0,224 pour les associations implicites), avancent que ces petits effets statistiques - obtenus le plus souvent dans un cadre expérimental - traduisent des effets importants dans la vie « réelle ». D'un autre côté, suite également à une méta-analyse de ces liens (Oswald, Mitchell, Blanton, Jaccard, Tetlock, 2013), Oswald et coll. (2015) considèrent au contraire que ces effets dans la vie « réelle » sont soit faibles soit, en l'état actuel des recherches, très difficiles à estimer et qu'il convient donc d'être très prudent quant à la généralisation à la réalité sociale des effets obtenus en laboratoire. D'après eux, tant qu'on ne disposera pas d'investigations longitudinales contrôlées dans les milieux où peuvent se produire de tels effets (écoles, entreprises, administrations, etc.), on restera dans une certaine indétermination quant à ces effets. En rappelant ces débats, il ne s'agit pas de nier que l'adhésion aux stéréotypes (Dovidio, Gaertner, 2000 ; Dovidio, Kawakami, Gaertner, 2002) et, plus spécifiquement, aux stéréotypes de sexe (Fiske, 1998) peut se traduire par des comportements discriminatoires, mais de s'interroger sur la généralité de ces effets et sur les problèmes posés par la généralisation à la vie réelle de résultats obtenus en laboratoire.

\section{CONCLUSION}

Si on fait la synthèse des différents points que nous avons évoqués, on peut dire que l'étude des 
stéréotypes de sexe soulève de nombreuses questions. Contrairement à ce que montrent fréquemment les campagnes de sensibilisation et nombre de travaux de psychologie sociale, les stéréotypes n'ont pas obligatoirement ce pouvoir qu'on leur attribue très souvent. D'abord, la perception et les jugements des individus ne sont pas nécessairement altérés par les stéréotypes de sexe. Ensuite, les mesures des stéréotypes de sexe ne sont pas nécessairement neutres et peuvent orienter vers une vision accentuant artificiellement la présence et le poids des stéréotypes. Enfin, la valeur prédictive (en termes de liens avec les comportements discriminatoires) des tests d'évaluation des stéréotypes, et notamment ceux de sexe, reste sujette à débat.

Plus fondamentalement, les études sur les stéréotypes, en particulier de sexe, posent à notre avis quatre problèmes. D'abord, elles envisagent souvent les stéréotypes comme une pensée qui serait fréquemment subie par les individus (Jussim, 2012). Or, c'est risquer de méconnaître le fait que les individus peuvent faire référence aux stéréotypes selon des niveaux de jugements et de perspectives différents, et qu'il faut prendre en compte l'interaction entre l'individu et les caractéristiques de la situation dans laquelle il se trouve (Fraisse, 2014 ; Sabatier, Schadron, Milhabet, Priolo, 2010 ; Yzerbyt, Judd, Corneille, 2004). Ainsi, les individus peuvent les utiliser, certes au premier degré, mais aussi en jouer de façon subtile, par exemple à titre humoristique ou de provocation dans une discussion. Ensuite, ces études qui ont pour objectif de lutter contre la discrimination, tendent implicitement à adopter un schéma causal univoque : ce seraient les stéréotypes qui provoqueraient des discriminations. Est-ce nécessairement le cas ? Comme l'a montré Sherif en son temps (1951), les stéréotypes peuvent être aussi une conséquence de la nature des relations objectives de discriminations entre groupes, ou les deux à la fois, causes et conséquences. Enfin, c'est tendre à mettre de côté le fait, qu'en dehors des situations expérimentales dans lesquelles on essaie de neutraliser un certain nombre de variables pour voir les effets spécifiques des stéréotypes de sexe que la procédure a activé, que dans les situations ordinaires, les individus sont constamment face à des dynamiques hétérogènes dont certaines peuvent s'opposer à ces stéréotypes. Pensons à l'enfant qui, à la fois, peut être amené à entendre des stéréotypes de sexe très traditionnels (par exemple quand on lui lit, avant de s'endormir, des contes issus des grands classiques de la littérature enfantine), constater des différences de comportements entre femmes et hommes en accord avec ces stéréotypes, mais remarquer, dans le même temps, que son père fait aussi la cuisine, le ménage, tandis que sa mère est active en dehors de la maison, qu'elle travaille, prend des décisions qui concernent la famille. Il faut être attentif au fait que de multiples influences, tantôt similaires, tantôt opposées, sont source de réflexion pour l'enfant et peuvent lui permettre, finalement, de prendre ses distances avec les stéréotypes traditionnels et de former son propre jugement (Fraisse, 2014 ; Rouyer, 2007).

En définitive, pour revenir à la question de la mobilisation vers plus d'égalité entre les sexes, il nous semble, qu'au lieu de se focaliser systématiquement sur le poids des stéréotypes de sexe (Whitley, Kite, 2010), ce qui comporte le risque de donner une vision figée des relations femmes/ hommes et de rendre les stéréotypes plus importants qu'ils ne sont, il convient, d'une part de montrer que nous ne sommes pas nécessairement victimes des stéréotypes, que nous pouvons faire appel à notre autonomie de raisonnement et, d'autre part, sans céder à un certain irénisme, de mettre en avant les situations où les stéréotypes de sexe, les menaces qu'ils représentent sont justement mis de côté dans les choix des individus (par exemple femme scientifique, femme P.D.G., homme sagefemme ou infirmier, assistant maternel, etc.). De mettre en avant, au bout du compte, les ressources des individus qui se saisissent des situations et les transforment pour devenir, chacun à leur niveau, uniques en leur genre (Rouyer, Mieyaa, le Blanc, 2014). Certes, nous ne sommes pas les premiers à évoquer ces inflexions. Simplement, cela ne nous paraît pas totalement inutile de les rappeler au vu de certaines orientations de la psychologie sociale des relations entre sexes, qui utilisent une lecture en termes de domination masculine. Ajoutons, qu'en deçà et au-delà des stéréotypes de sexe, ce sont les situations concrètes, matérielles, dans lesquelles les individus et les groupes vivent, qui favorisent dévalorisation de soi, fausse-conscience, stéréotypes, préjugés et discriminations (Sherif, Sherif, 1969). C'est la modification de ces conditions concrètes d'existence, faites d'inégalités et d'injustices, qui constitue une priorité majeure.

Cela dit, on peut se demander les raisons de cet écart entre l'importance accordée aux stéréotypes de sexe par nombre de travaux en psychologie sociale et leurs effets parfois ambigus quand on les confronte à la réalité sociale qu'ils sont censés dépeindre. Si l'attention aux stéréotypes de sexe résulte, à la fois, d'une longue et fructueuse tradition d'étude des stéréotypes en psychologie sociale et de 
la volonté légitime de lutter contre les discriminations, la tendance à la surestimation des effets attribués aux stéréotypes de sexe tient à l'idée que nous en serions les victimes involontaires, voire actives. Cette idée relève maintenant du sens commun et des politiques gouvernementales (Naves, Wisnia-Weill, 2014). Il y a ainsi adéquation entre les attendus de la discipline et la demande sociale qui favorise l'attention et l'orientation sur les aspects négatifs des stéréotypes. Cette manière de voir s'explique, à notre avis, par le fait que nombre de travaux s'inscrivent dans le cadre des théories de la domination notamment masculine ${ }^{15}$. Ces théories mettent en avant que nos structures mentales sont largement déterminées, altérées de façon inconsciente par les positions sociales différentes des femmes et des hommes, que l'idéologie légitimant la domination masculine, et que véhiculeraient les stéréotypes de

15. Quant à cette « domination » des théories de la domination masculine dans le champ des études sur le genre, on peut l'expliquer par la référence à ce qui en fut à l'origine : les mouvements féministes des années 1960 et 1970, dont l'un des objectifs principaux était la lutte nécessaire contre la domination masculine (Fraisse, 2014 ; Froidevaux-Metterie, 2015) sexe, s'inscrit au cœur de nos modes d'appréhension du réel (Bourdieu, 1998 ; Jost, Banaji, 1994 ; Lorenzi-Cioldi, 2009 ; Sidanius, Pratto, 1999). Ce point de vue nous paraît problématique car il ne tient pas compte des évolutions sociales vers plus d'égalité entre les sexes et des capacités d'autonomie des acteurs sociaux. Dans un prochain article (Tostain, à paraître), nous nous interrogerons sur ces théories psychosociales de la domination. Nous évoquerons des données empiriques et expérimentales issues de travaux sociologiques et psychologiques qui questionnent et vont à l'encontre des attendus issus d'une vision en termes de domination masculine, et nous soulignerons certains problèmes associés aux postulats de ces théories. Enfin, nous nous attacherons à présenter d'autres approches des relations entre sexes qui, en mettant l'accent sur les dynamiques d'appropriation des sujets, nous semblent à la fois plus respectueuses des acteurs sociaux, plus susceptibles de rendre compte des évolutions sociales actuelles entre femmes et hommes - et notamment de la pluralité des situations des unes et des autres et de la variabilité intrasexe -, et qui, finalement, nous paraissent plus pertinentes pour aller vers plus d'égalité entre les premières et les seconds.

\section{RÉFÉRENCES}

AlaPhILIPPE (Daniel).- L'imposteur auto-mystifié, Bulletin de psychologie, 39, 3-6, n 374, 1986, p. 191-196.

Allport (Gordon W.).- The Nature of Prejudice, New York, Doubleday Anchor books, 1954.

Arkes (Hal R.), Tetlock (Philip E.).- Attributions of implicit prejudice, or "Would Jesse Jackson 'fail' the implicit association test?", Psychological Inquiry, 15, 4, 2004, p. 247-278.

BAdinter (Élisabeth).- Fausse route, Paris, Odile Jacob, 2003.

Beauvorr (Simone de).- Le deuxième sexe, Paris, Gallimard, 1949.

BEM (Sandra L.).- The measurement of psychological androgyny, Journal of Consulting and Clinical Psychology, 42, 1974, p. 155-162.

BEM (Sandra L.).- The BSRI and gender schema Theory: A reply to Spence and Helmreich, Psychological Review, 88, 4, 1981, p. 369-371.
Blaison (Christophe), Chassard (Delphine), Kop (JeanLuc), GANA (Kamel).- L'IAT (Implicit Association Test) ou la mesure des cognitions sociales implicites : revue critique de la validité et des fondements théoriques des scores qu'il produit, L'Année psychologique, 106, 2006, p. 305- 336.

Bonnot (Virginie), Croizet (Jean-Claude).- Stereotype threat and stereotype endorsement: Their joint influence on mathematic performance, Revue internationale de psychologie sociale, 24, 2, 2011, p. 105-120.

Bourcier (Marie-Hélène), Moliner (Alice).-Comprendre le féminisme : essai graphique, Paris, Max Milo, 2012.

Bourdieu (Pierre).- La domination masculine, Paris, Seuil, 1998.

Bourhis (Richard Y.), LeYens (Jacques-Philippe).Stéréotypes, discrimination et relations intergroupes, Bruxelles, Mardaga, 1998.

BRANDT (Mark J.).- Sexism and gender inequality across 57 societies, Psychological Science, 22, 2011, p. 1413-1418. 
BREDA (Thomas), Ly (Son T.).- Professors in core science fields are not always biased against women: Evidence from France, Paris School of Economics-CNRS, 2014, p. 1-40.

Castel (Pierre-Henri).- La métamorphose impensable. Essai sur le transsexualisme et l'identité personnelle, Paris, Gallimard, 2013.

Chor (Namok), Fuqua (Dale R.), Newman (Jody L.).- The Bem sex-role inventory: Continuing theorical problems, Educational and Psychological Measurement, 68, 5, 2008, p. 881-900.

Condry (John), Condry (Sandra).- Sex differences: A study of the eye of the beholder, Child Development, 47, 1976, p. 812-819.

Croizet (Jean-Claude), Claire (Theresa).- Extending the concept of stereotype threat to social class: The intellectual underperformance of students from low socioeconomic background, Personality and Social Psychology Bulletin, 24, 6, 1998, p. 588-594.

Croizet (Jean-Claude), Dutrévis (Marion).Socioeconomic status and intelligence: Why test scores do not equal merit, Journal of Poverty, 8, 2004, p. 91-107.

Dardenne (Benoit), Delacollette (Nathalie), Grégoire (Christine), LeCoce (Delphine).- Structure latente et validation de la version française de l'Ambivalent Sexism Inventory : l'échelle de sexisme ambivalent, L'Année psychologique, 196, 2006, p. 235-264.

Dardenne (Benoit), Dumont (Muriel), Bollier (Thierry).Insidious dangers of benevolent sexism: Consequences for women's performance, Journal of Personality and Social Psychology, 93, 5, 2007, p. 764-779.

Deaux (Kay), Emswiller (Tim).- Explanation of successful performance on sex-linked tasks: What is skill for the male is luck for the female, Journal of Personality and Social Psychology, 29, 1, 1974, p. 80-85.

Dovidio (John F.), GAERTNER (Samuel L.).-Aversive racism and selection decisions: 1989 and 1999, Psychological Science, 11, 4, 2000, p. 315-319.

Dovidio (John F.), Kawakami (Kerry), Gaertner (Samuel L.).- Implicit and explicit prejudice and interracial interaction, Journal of Personality and Social Psychology, 82, 1, 2002, p. 62-68.

Eagly (Alice H.), Mladinic (Antonio).- Are people prejudiced against women? Some answers from research on attitudes, gender stereotypes, and judgments of competence, dans Stroebe (W.), Hewstone (M.), European Review of Social Psychology, 5, Londres, Wiley, 1994, p. 1-35.

Eagly (Alice H.), Mladinic (Antonio), Otto (Stacey).Are women evaluated more favorably than men? An analysis of attitudes, beliefs and emotions, Psychology of Women Quarterly, 1, 2, 1991, p. 203-216.

Fiske (Susan T.).- Prejudice, stereotyping and discrimination, dans Gilbert (D .T.), Fiske (S. T.), Lindzey (G.), The Handbook of Social Psychology, New York,
McGraw-Hill, 1998, p. 357-411.

Fontayne (Paul), Sarrazin (Philippe), Famose (JeanPierre).- The Bem sex-role Inventory: Validation of short version for French teenagers, European Review of Applied Psychology, 50, 4, 2000, p. 405-416.

FouQue (Antoinette).- Il y a deux sexes : essais de féminologie, Paris, Folio, 2015.

Fraisse (Geneviève).- La différence des sexes, Paris, Presses universitaires de France, 1996.

Fraisse (Geneviève).- Les excès du genre. Concept, image, nudité, Paris, Lignes, 2014.

Froidevaux-Metterie (Camille).- La révolution $d u$ féminin, Paris, Gallimard, 2015.

Gana (Kamel).- Androgynie psychologique et valeurs socio-cognitives des dimensions du concept de soi, Les cahiers internationaux de psychologie sociale, 25, 1995, p. 27-43.

Ganley (Colleen M.), Mingle (Leigh A.), Ryan (Allison M.), RyAn (Khaterine), VAsilyeva (Marina), Perry (Michelle).-An examination of stereotype threat effects on girl's mathematics performance, Developmental Psychology, 49, 10, 2013, p. 1886-1897.

Gergen (Kenneth).- Social Psychology as History, Journal of Personality and Social Psychology, 26, 1973, p. 309-320.

Gilligan (Carol).- Une si grande différence, Paris, Flammarion, 1986.

GLICK (Peter), FISKE (Susan T.).- The ambivalent sexism inventory: Differentiating hostile and benevolent sexism, Journal of Personality and Social Psychology, 70, 3, 1996, p. 491-512.

GoldBerg (Philip).- Are women prejudiced against women? Trans-Action, 5, 5, 1968, p. 28-30.

Gough (Harrison G.).- Manual for the California Psychological Inventory, Palo Alto, California, Consulting psychologists press, 1957.

Greenwald (Antony G.), Banaji (Mahzarin R.), NoseK (Brian A.).- Statistically small effects of the Implicit association test can have societally large effects, Journal of Personality and Social Psychology, 108, 2015, p. 553-561.

Greenwald (Anthony G.), McGhee (Debbie E.), Schwartz (Jordan L. K.).- Measuring individual differences in implicit cognition: The Implicit association test, Journal of Personality and Social Psychology, 74, 1998, p. 1464-1480.

Greenwald (Anthony G.), Poehlman (T. Andrew), Uhlmann (Eric L), BANAJI (Mahzarin R.).- Understanding and using the Implicit association test. III. Meta-analysis of predictive validity, Journal of Personality and Social Psychology, 97, 1, 2009, p. 17-41.

HАвів (Claude).- La galanterie française, Paris, Gallimard, 2006. 
Haccoun (Dorothy M.), Stacy (Stanley).- Perceptions of male and female success or failure in relation to spouse encouragement and sex-association of occupation, Sex Roles, 6, 1980, p. 819-832.

Hirata (Helena), Laborie (Françoise), Le Doaré (Hélène), SEnotier (Danièle).- Dictionnaire critique du féminisme, Paris, Presses universitaires de France, 2000.

Huguet (Pascal), RÉGner (Isabelle).- Stereotype threat among schoolgirls in quasi-ordinary classroom circumstances, Journal of Educational Psychology, 99, 3, 2007, p. 545-560.

Huguet (Pascal), RÉGNER (Isabelle).- Counter-stereotypic beliefs in math do no protect school girls from stereotype threat, Journal of Experimental Social Psychology, 45, 2009, p. 1024-1027.

Irigaray (Luce).- Je, tu, nous. Pour une culture de la différence, Paris, Grasset, 1992.

Jost (John T.), BanaJI (Mahzarin R.).- The role of stereotyping in system justification and the production of false-consciousness, British Journal of Social Psychology, 33, 1994, p. 1-27.

Jost (John T.), BANAJI (Mahzarin R.), Nosek (Brian A.).- A decade of system justification theory: Accumulated evidence of conscious and unconscious bolstering of the statu quo, Political Psychology, 25, 6, 2004, p. 881-919.

Jussim (Lee)-- Social Perception and Social Reality: Why Accuracy Dominates Bias and Self-Fulfilling Prophecy, New York, Oxford university press, 2012.

Karpinski (Andrew), Hilton (James L.).- Attitudes and the implicit association test, Journal of Personality and Social Psychology, 81, 2001, p. 774-788.

Katz (Irvin), Epps (Edgar G.), Axelson (Leland J.).Effect upon Negro digit symbol performance of comparison with Whites and with other Negroes, Journal of Abnormal and Social Psychology, 69, 1964, p. 963-970.

K’Delant (Pascaline), Gana (Kamel).- Analyse multitraits-multiméthodes des scores au questionnaire d'attributs personnels (Personal attributes questionnaire [PAQ]) auprès d'un échantillon féminin, Psychologie française, 54, 2009, p. 323-329.

Kaufmann (Jean-Claude).- Sociologie du couple, Paris, Presses universitaires de France, 2014

Kray (Laura J.), Thompson (Leigh), Galinsky (Adam).Battle of the sexes: Gender stereotype confirmation and reactance in negotiations, Journal of Personality and Social Psychology, 80, 6, 2001, p. 942-958.

LauRens (Stéphane), Moscovici(Serge).-The confederate's and others' self-conversion: A neglected phenomenon, The Journal of Social Psychology, 145, 2, 2005, p. 191-207.

LÉGAl (Jean-Baptiste), Delouvée (Sylvain).- Stéréotypes, préjugés et discriminations, Paris, Dunod, 2008.
Levenson (Hanna), Burford (Brent), Bonno (Bobbie), DAvis (Loren).- Are women still prejudiced against women? A replication and extension of Goldberg's study, The Journal of Psychology, 89, 1, 1975, p. 67-71.

Lindberg (Sarah M.), Hyde (Janet S.), Petersen (Jennifer L.), LinN (Marcia C.).-New trends in gender and mathematics performance: A meta-analysis, Psychological Bulletin, 136, 6, 2010, p. 1123-1135.

Lippmann (Walter).- Public Opinion, New York, Harcourt, Brace and Co, 1922.

Lips (Hilary M.), Myers (Anita M.).- Subject reactions to a stimulus person as a function of sex of subject and sex-role appropriateness of stimulus person's career goal, Sex Roles, 6, 1980, p. 675-682.

Locksley (Anne), Colten (Mary E.).- Psychological Androgyny: A case of mistaken identity?, Journal of Personality and Social Psychology, 37, 6, 1979, p. 1017-1031.

LORENZI-CIOLDI (Fabio).- Les représentations des groupes dominants et dominés : collections et agrégats, Grenoble, Presses universitaires de Grenoble, 2009.

Lynott (Dermott), Connell (Louise), O'Brien (Kerry S.), Kansal (Himanshu).- Modeling the IAT: Implicit association test reflects shallow linguistic environment and not deep personal attitudes, dans Miyake (N.), Peebles (D.), Cooper (R.P.), Proceedings of the $34^{\text {th }}$ Annual Conference of the Cognitive Science Society, Austin, TX, Cognitive science society, 2012, p. 1948-1953.

McConnell (Allen R.), Leibold (Jill M.).- Relations among the implicit association test, discriminatory behavior, and explicit measures of racial attitudes, Journal of Experimental Social Psychology, 37, 2001, p. 435-442.

Ministère de L'ÉduCATION NATIONALE.- Filles et garçons : sur le chemin de l'égalité, 2013.

Morchain (Pascal), Schadron (Georges), Saint-Bauzel (Roxane), BÉCHU (Géraldine). - « Le parfum ». Quand l'odeur d'un candidat affecte le recruteur... ou pas : une question de déterminabilité, Revue Internationale de psychologie sociale, 26, 2, 2013, p. 73-79.

Naves (Marie-Cécile), Wisnia-Weill (Vanessa).- Lutter contre les stéréotypes filles-garçons, Commissariat général à la stratégie et à la prospective, janvier, 2014.

ORNE (Martin T.).- On the social psychology of the psychological experiment: With particular reference to demand characteristics and their implications, American Psychologist, 17, 11, 1962, p. 776-782.

Osgood (Charles E.), Suci (George J.), Tannenbaum (Percy H.).- The Measure of Meaning, Urbana, University of Illinois, 1957.

Oswald (Frederick L.), Mitchell (Gregory), Blanton (Hart), JACCARD (James), Tetlock (Philip E.).- Predicting Ethnic and racial discrimination: A meta-analysis of IAT criterion studies, Journal of Personality and Social Psychology, 105, 2, 2013, p. 171-192. 
Oswald (Frederik L.), Mitchell (Gregory), Blanton (Hart), JacCARd (James), TetLock (Philip E.).- Using the IAT to predict ethnic and racial discrimination: small effect sizes of unknown societal significance, Journal of Personality and Social Psychology, 108, 4, 2015, p. 562-571.

Post (Robin D.).- Causal explanations of male and female academic performance as a function of sex-role biases, Sex Roles, 7, 7, 1981, p. 691-698.

RÉGNER (Isabelle), SteEle (Jennifer R.), Ambady (Nalini), Thinus-Blanc (Catherine), Huguet (Pascal).- Our future scientists: A review of stereotype threat in girls from early elementary school to middle school, Revue internationale de psychologie sociale, 27, 3, 2014, p. 13-51.

Revue EsPrit.- Les controverses du féminisme, Esprit, n ${ }^{\circ} 398$, octobre 2013.

Riecken (Henry W.).- A program for research on experiments in social psychology, dans Washburne (N. F.), Decisions, Values and Groups, Oxford, Pergamon press, 1962, p. 25-41.

Rosenthal (Robert).- The effect of the experimenter on the results of the psychological research, dans Maher (B. A.), Progress in Experimental Personality Research, New York, Academic press, 1964, p. 79-114.

Rouyer (Véronique), MieyaA (Yoan), Le Blanc (Alexis).Socialisation de genre et construction des identités sexuées : contextes sociétal et scientifique, acquis de la recherche et implications pratiques, Revue française de pédagogie, $\mathrm{n}^{\circ} 187,2014$, p. $97-137$.

Sabatier (Candy), Schadron (Georges), Milhabet (Isabelle), Priolo (Daniel).- La déterminabilité de « celui qui juge »: impact sur l'orientation d'un jugement et sur la formation d'impression, L'Année psychologique, 110, 4, 2010, p. 573-593.

SACKetT (Paul R.), Hardison (Chaitra M.), Cullen (Michael J.).- On interpreting stereotype threat as accounting for African American-White differences on cognitive tests, American Psychologist, 59, 1, 2004, p. 7-13.

Sarlet (Marie), Dardenne (Benoit).- Le sexisme bienveillant comme processus de maintien des inégalités sociales entre les genres, L'Année psychologique, 112, 2012, p. 435-463.

Schadron (Georges).- Déterminabilité sociale et essentialisme psychologique : quand une conception essentialiste renforce la confirmation des attentes stéréotypiques, Les cahiers internationaux de psychologie sociale, 67-68, 3, 2005, p. 77-84.

SHERIF (Muzafer).- A preliminary experimental study of inter-group relations, dans Rohrer (J. H.), Sherif (M.), Social Psychology at the Crossroads, New York, Harper and Row, 1951, p. 388-424.
SHERIF (Muzafer), SHerif (Carolyn W.).- Social Psychology, New York, Harper et Row, 1969.

Shin (Margaret), PitTinsky (Todd L.), Ho (Geoffrey C.).Stereotype boost: Positive outcomes from the activation of positive stereotypes, dans Inzlicht (M.), Schmader (T.), Stereotype Threat: Theory, Process, and Application, Oxford, university press, 2012, p. 141-156.

Sidanius (Jim), Pratto (Felicia).- Social Dominance: An Intergroup Theory of Social Hierarchy and Oppression, Cambridge, Cambridge university press, 1999.

Sidanius (Jim), Pratto (Felicia), LaAr van (Colette), Levin (Shana).- Social dominance theory: Its agenda and method, Political Psychology, 25, 6, 2004, p. 845-880.

SingLY (François de).-Le soi, le couple et la famille, Paris, Armand Colin, 2005.

Smith (Stephanie H.), Whitehead (George I.), Sussman (Nan M.).- Perception of female and male success in United States and Third World nations, Sex Roles, 10, 11-12, 1984, p. 903- 911.

STeEle (Claude M.), Aronson (Joshua).- Stereotype threat and the intellectual test performance of African Americans, Journal of Personality and Social Psychology, 69, 5, 1995 , p. 797-811.

STERn (Marilyn), KarRaker (Katherine H.).- Sex stereotyping of infants: A review of gender labeling studies, Sex Roles, 20, 1989, p. 501-522.

Steuer (Faye B.), Bode (Blair C.), Rada (Kelley E.), Hittner (James B.).- Gender labeling and perceived infant emotionality: A partial replication of a "classic" study, Psychological Reports, 107, 1, 2010, p. 139-144.

Swim (Janet), Borgida (Eugene), Maruyama (Geoffrey), Myers (David G.).- Joan McKay versus John McKay: Do gender stereotypes bias evaluation?, Psychological Bulletin, 105, 1989, p. 409-429.

Swim (Janet K.), SANnA (Lawrence J.).- He's skilled, she's lucky: A meta-analysis of observers' attributions for women's and men's successes and failure, Personality and Social Psychology Bulletin, 22, 5, 1996, p. 507-519.

TERrier (Camille).- Giving a little help to girls? Evidence on grade discrimination and its effets on students achievement, Paris School of Economics-CNRS, 36, 2014, p. 1-41.

Tetlock (Philip E.), Mitchell (Gregory).- Calibrating prejudice in milliseconds, Social Psychology Quarterly, 71, 1, 2008, p. 12-16.

THÉRY (Irène).- La distinction de sexe : une nouvelle approche de l'égalité, Paris, Odile Jacob, 2007.

Tisserant (Pascal), Bourhis (Richard Y.).- Discrimination et gestion de l'égalité et de la diversité, Université de Lorraine, canal U, 2013. 
Tostain (Manuel).- Pour en finir avec la domination masculine ? Regard critique sur les approches psychosociales des relations entre sexes, Bulletin de psychologie, à paraître.

Tougas (Francine), Brown (Rupert), Beaton (Ann M.), Joly (Stéphane).- Neosexism: Plus ça change, plus c'est pareil, Personality and Social Psychology Bulletin, 21, 8, 1995, p. 842-849.

UlLrich (Johannes), SCHLÜTER (Elmar).- Detecting nasty data with simple plots of complex models: Comments on Brandt (2011), Psychological Science, 23, 7, 2012, p. 824-825.

VAn Loo (Katie J.), Rydell (Robert J.).- On the experience of feeling powerful: Perceived power moderates the effect of stereotype threat on women's math performance, Personality and Social Psychology Bulletin, 39, 3, 2013, p. 387-400.

Verniers (Catherine), Martinot (Delphine).- L'EJSAG, une échelle de justification du système spécifique au genre : validation auprès d'une population d'adolescents et d'adultes, L'Année psychologique, 115, 1, 2015, p. 107-140.

Whitley (Bernard E.), Kite (Mary E.).- The Psychology of Prejudice and Discrimination, Belmont, CA, Wadsworth, 2010.

Yzerbyt (Vincent), Judd (Charles M.), Corneille (Olivier).- Psychology of Group Perception, New York, Psychology press, 2004. 